\title{
Surgical management of a rare case of giant penile cancer.
}

$\underline{\text { Campi }}^{1}$, $\underline{\text { Sessa }}^{1}, \underline{\text { Cocci }}^{2}, \underline{\text { Sforza }}^{1}, \underline{\text { Greco }}^{1}, \underline{\text { Cito }}^{1}, \underline{\text { Vanacore }}^{1}$, Raspollini MR ${ }^{3}$, $\underline{\text { Serni }}^{1}$, Lapini $\underline{A}^{1}$, Carini $M^{1}$, Minervini $A^{1}$.

\section{Author information}

\section{Abstract}

Primary penile cancer is a rare malignant disease. In most cases, it presents as a clinically obvious lesion leading to early diagnosis in most patients. However, even in developed Countries, it carries a significant social stigma leading to diagnosis at locally advanced stages in a non-negligible proportion of patients. Yet, bulky penile lesions are becoming extremely rare in current clinical practice. We present a case of a patient with a giant primary penile cancer managed with radical penectomy, bilateral inguinal lymphadenectomy and perineal urethrostomy, who experienced disease recurrence 6 months after surgery and died with metastatic disease after denial of further treatment. The management of our case was challenging due to the extremely late diagnosis, the huge dimensions and the infiltrative nature of the tumour; however, from a histopathological perspective, the cancer itself did not display any microscopic peculiarity. Our case highlights that such bulky penile tumors can still occur in current urologic practise and require complex salvage surgical interventions in the context of a multidisciplinary setting. 